\title{
From physics to art and back
}

Marco Leona ${ }^{1}$, Kaori Fukunaga ${ }^{2}$, Haida Liang ${ }^{3}$, Piero Baglioni ${ }^{4}$, Giulia Festa ${ }^{5}$, Vladimir Levchenko ${ }^{6}$

1 Department of Scientific Research, The Metropolitan Museum of Art, New York, NY, 10028, USA

2 National Institute of Information and Communications Technology (NICT), Koganei, Tokyo, Japan

3 School of Science and Technology, Nottingham Trent University, Nottingham NG11 8NS, UK

4 Consorzio Interuniversitario per lo Sviluppo dei Sistemi a Grande Interfase (Center for Colloid and Surface Science), CSGI, and Chemistry Department, University of Florence, 50019, Florence, Italy

5 Museo Storico Della Fisica e Centro Studi e Ricerche "Enrico Fermi", Rome, Italy

6 Australian Nuclear Science and Technology Organisation (ANSTO), Kirrawee DC, New South Wales, Australia

Email: Marco.Leona@metmuseum.org, kaori@nict.go.jp, haida.liang@ntu.ac.uk, baglioni@csgi.unifi.it, giulia.festa@cref.it, vld@ansto.gov.au

Standfirst: Scientists studying cultural heritage use a variety of physics techniques to understand how pieces were made, their history and how to best preserve them. Six scientists who use different techniques describe their work - and how working with cultural heritage can lead to physics developments, too.

\section{[H1] How can we learn from old pigments?}

\section{Marco Leona}

As a scientist working in an art museum, I investigate the material aspects of works of art. By applying physics and chemistry techniques to works by artists of every culture, time and place, my colleagues and I seek to build a material history of art: a connection between materials and meaning. We tell stories about art and the people who make it, highlighting cultural practices, economic and social relationships, technical developments, spiritual insights or imminent vulnerability. We are not only scientists but also historians, and if the works of art are our primary sources, scientific techniques are the tools we use to uncover evidence and build connections. At the same time, the analytical challenges posed by studying artists' materials and techniques can stimulate scientific discoveries.

I first saw Hokusai's Great Wave (Fig. 1a) as a child, as a postage stamp-sized illustration I found while leafing through a compact encyclopedia of art. Decades later, I would hold in my hands the best extant copy of this iconic print, to study it by fiber optics reflectance spectroscopy (FORS) and hyperspectral 
imaging. The striking blue of the ocean and the vertiginous sense of depth one experiences in looking at the hollow of the Wave come from Prussian blue. Bright and saturated even at full density, cheap and easy to use, this synthetic pigment revolutionized Japanese woodblock printing in the 1830s [1]. Yet, the anonymous printers of The Great Wave did not simply substitute Prussian blue for the more traditional (and duller) indigo. Spectroscopic analysis shows that they mixed the two blues to expand the tonal range and darken the hue without reducing its saturation.

Beyond revealing the past, the scientific study of art offers challenges to applied science, and I would encourage my colleagues in physics labs over the world to turn to art for practical inspiration. Fifteen years ago I started using surface-enhanced Raman scattering (SERS) to solve one of the most difficult problems in art analysis: identifying organic dyes. These are molecules extracted from plants or insects that have strong coloring power, and have been used the world over for millennia to dye textiles and to produce pigments. Using silver nanoparticles to increase the intensity of Raman scattering from the small amount of dye molecules, exploiting a process called plasmon resonance, we have been able to achieve unprecedented sensitivity [2]. Over the years several other laboratories in universities and museums have joined this effort.

\section{[H1] How can we see the layers of a painting and plan its conservation?}

\section{Kaori Fukunaga}

For many in the general public, the scientific study of art is associated with the determination of materials used by artists and the detection of hidden drawings under famous paintings. However, investigations on the internal observation of preparation layers and supports are also important for conservation planning, like a careful examination before medical surgery.

Because artworks are unique, heritage scientists seek measurement techniques that are nondestructive and even that do not involve contact with the object under study. Electromagnetic techniques meet this requirement and are suitable for observing internal structures.

The THz time-domain imaging system irradiates ultrashort pulsed waves from the surface and detects reflection pulse sequences that correspond to the internal layer structure. A typical commercial system enables observations up to approximately $5 \mathrm{~mm}$ from the surface with a resolution of approximately 0.3 $\mathrm{mm}$. As the preparation layers of most paintings are less than $5 \mathrm{~mm}$, a detailed layer structure can be observed.

By scanning the object with an X-Y or 3D scanning system, internal structures of the preparation and supporting layers can be visualized as not only cross-section images at any point, but also spatial images at a given depth, as if peeling the object from the surface. These results provide valuable information [3]. In the Polittico di Badia by Giotto di Bondone, painted around 1300, the methods revealed that Giotto used a medieval technique, using two gesso layers in the panel, with a cloth placed between them. 
In some cases, the results of measurements have led to important discoveries in art history. For example, "Irises at Yatsuhashi" by Ogata Kōrin (Fig. 1b), dating from the early 1700s, is one of the most famous Japanese panel screens with gold background. It was found to be entirely covered with gold leaf on the base, unlike other works from the time. THz time-domain imaging revealed a new painting technique developed by the artist and his school. Applying the gold leaf uniformly over the entire surface of the painting is easier than matching it to the design. However, it is not easy to paint directly on the gold leaf. Kōrin developed a new painting technique on gold leaf by varying the particle size of the pigments. It allowed the school to produce more artworks than before.

The outcomes of these discoveries cannot be measured in terms of economic value, as the findings and research records become part of history along with the artworks over the centuries.

\section{[H1] How has studying cultural heritage lead to advances in optical coherence tomography?}

\section{Haida Liang}

To conserve a cultural object - whether it's an old master painting (Fig. 1C), ceramic or piece of carved jade - it is important to understand its cross-section and layers of materials before attempting restoration. The cross-sections of objects also give information about their history and how they were made. Traditionally, only very small samples could be taken from edges or existing cracks on certain types of objects to have their cross-section examined under a microscope, meaning that most or all of the object would go unmapped. But by the mid-2000s, optical coherence tomography (OCT) came on the scene, enabling non-contact and non-invasive imaging that could be applied anywhere on an object or even an entire object.

OCT was developed thanks to technological advances in fibre-based broad-band lasers and fast computers, and was first used for in vivo imaging of the interior of the eye. It is a fast scanning Michelson interferometer for 3D imaging of surface and subsurface microstructure. Unlike confocal microscopy where the $3 \mathrm{D}$ resolution is determined by the objective lens such that high depth resolution means small field of view (typically less than $1 \mathrm{~mm}$ ), OCT axial resolution (in the depth direction) is given by the bandwidth of the laser, which is decoupled from its transverse resolution given by the objective lens, allowing for high-depth-resolution imaging of thin layers with a wide field of view.

Applying OCT to heritage materials has required the development of new instruments, owing to the heterogeneity and complexity of the materials under study. For example, OCT for biomedical imaging normally operates in the near-infrared at $800 \mathrm{~nm}$ or $1300 \mathrm{~nm}$, where water absorption is minimal. However, polychrome objects derive their colour from being either highly scattering or absorbing in parts of the visible spectrum. For instance, in ancient Egyptian faience, the copper oxide in the glaze absorbs strongly around $800 \mathrm{~nm}$, which limited the probing depth. Such difficulties led to the development of longwavelength OCT at 2 microns [4,5], which offers the best penetration depth in materials with low water content. Suitable broadband laser sources at 2 microns also had to be developed, to give the required 
depth resolution. Nobody could have predicted that the Michelson interferometer - made at the end of the $19^{\text {th }}$ century for fundamental physics research, showing that the speed of light is invariant of reference frames - could have made an impact in the conservation of cultural heritage and arts and humanities research more than 100 years later.

\section{[H1] How can soft-matter physics help us clean artwork safely and effectively?}

\section{Piero Baglioni}

How to best clean artifacts is an open and challenging issue in conservation science. Given the singular nature of iconic artworks, and the large number of objects in collections worldwide, it is essential to find effective, safe and time-efficient cleaning tools. Conservation practice traditionally relies on classic solvent and polymer chemistry, but these can lack adequate control or selective removal of unwanted layers, especially on the solvent-sensitive artifacts frequently met in modern and contemporary art. Soft-matter physics is the ideal framework to develop advanced cleaning systems that cope with these challenges.

For instance, selective detachment of soil and coatings can be aided by cleaning fluids designed to retract from a surface and form beads, a process known as de-wetting. Diffusivity in gels is another example, where knowledge of transport processes in soft matter makes it possible to capture dirt and control the wetting of water-sensitive and solvent-sensitive surfaces, without risks for the artifacts [6].

Twin-chain polymer networks (TC-PNs) [7] have been developed to control and tailor the diffusivity of solvents or complex fluids and transport processes in gels. They represent a breakthrough solution in cleaning art. In TC-PNs, two types of polyvinyl alcohol (PVA) - differing in molecular weight and how prone their bonds are to being broken by water - are blended together. Interpenetrating the two PVAs leads to a gel system that is better than traditional cleaners at dirt capture and transport and at controlled wetting at the gel-artefact interface, enabling selective detachment of dirt. In addition, the characteristics of TC-PCNs make them materials of interest to other fields such as drug-delivery, biology, tissue engineering and cosmetics.

Finally, complex fluids tailored to perform selectively a specific action (solubilization or de-wetting of a target molecule/polymer in a coating) can be loaded in the TC-PNs to give one of the most sophisticated cleaning tools available to conservators. These systems were successfully used for the restoration of works of art by Pablo Picasso, Jackson Pollock and Roy Lichtenstein, among others (Fig. 1d).

\section{[H1] How can we see the internal structure and composition of 3D objects?}

\section{Giulia Festa}


Is it possible to identify the contents of a sealed Egyptian vase without opening it? Or to discover the internal composition of weapons from the Bronze Age, revealing how they were forged? These are the kinds of question neutron methods can help answer in non-destructive and non-invasive ways, providing information that other techniques can't.

There is an extensive set of neutron techniques available for cultural heritage [8]. Many results have been obtained in multidisciplinary studies that provide information essential for setting proper restoration and conservation strategies, performing correct classification and dating of objects and understating the historical and anthropological settings of archaeological findings. In general, neutrons are able to penetrate thick layers without substantial attenuation, making them ideal to study the interior (bulk) properties of certain materials such as metals, pottery and stone. Additionally, the neutron probe features high sensitivity to light elements (mainly hydrogen). These unique combination of characteristics are extremely useful when studying sealed objects which contain organic materials, rich in hydrogen.

For example, we have used neutrons to study sealed Egyptian alabaster and ceramic vases [9], part of the Kha and Merit grave goods preserved at Museo Egizio in Turin, Italy (Fig. 1e). Neutron imaging allowed us to 'look inside' the vases, without opening them, and discover their internal morphology as well as the characteristics and shape of their contents, identified as organic materials; isotopic analysis revealed an abundance of different isotopes in the contents. Compared to other techniques, the possibility of revealing all such information in a totally non-destructive and non-invasive manner is invaluable when studying this kind of unique archaeological artifact.

As a physicist, working in interdisciplinary research is extremely stimulating and a truly enriching experience as it provides the opportunity to collaborate with researchers from many different fields, countries and background. Cultural heritage, in particular, brings together many different disciplines, methods, points of view and approaches. Through neutron techniques we provide accurate quantitative information which is often the starting point for wider research based on constant interaction and collaboration with archaeologists, paleontologists, historians, collection holders, restorers and curators. This kind of successful, active collaboration within cultural heritage can hopefully stimulate similar initiatives in other fields.

\section{[H1] How can we measure the age of ancient artwork?}

\section{Vladimir Levchenko}

It is common for new methods from material sciences, especially those that are non-destructive and versatile, to be soon applied to investigations of various cultural objects. Radiocarbon dating occupies a special place in the list of techniques. It is one of a few methods that provides temporal information 
about the object of research, making it possible to build chronologies, and to use art and cultural objects to shed light on human societies at different times.

The radiocarbon method itself emerged and was developed relying on ancient heritage objects - in the 1940s, Willard Libby, Nobel Prize winner for radiocarbon, used Pompeiian, Biblical and ancient Egyptian artifacts of known age to validate the ages estimated by radiocarbon and to check the half-life of ${ }^{14} \mathrm{C}$. However, when it first emerged, radiocarbon was not widely applied to cultural heritage objects per se directly. It is essentially a destructive method, and initially required grams of carbon for measurements. But with the advent of AMS (accelerator mass spectrometry) in the 1970s, sample sizes were reduced to a few milligrams of carbon, opening applications to many objects and studies not previously considered suitable. Further sophistication reduced size requirements to the microgram range, allowing dating of individual chemical constituents of the object [10]. The doors for cultural heritage studies were thrown open.

AMS radiocarbon dating of rock and cave art started soon after the technique appeared. Initially applications mainly concerned the prominent well-preserved charcoal drawings, where enough carbon material could be obtained without damaging the object. However, most rock drawings around the world were made with mineral pigments in rather open environments, resulting in poor preservation, with little or no carbon left to be analysed. When I was approached by archaeologists seeking help in radiometric dating of Australian rock art in the Arnhem Land (Fig. 1f) and later in the Kimberley region, it was a challenge to invent a procedure to obtain a reliable age estimate at all. Fortunately, we developed some approaches to reliably extract some datable carbon constituents from various mineral crusts and mud wasp nest remnants on rock walls. This, when coupled with our microsample capabilities, started producing dates, proving the Pleistocene antiquity of Australian Rock Art similar to the other regions of the world [11]. We physicists really enjoyed this work and appreciated the importance of evidencing the very ancient and common emergence of this unique human trait of artistic reflection of the surrounding world.

\section{References:}

1. Hokusai and the Blue Revolution in Edo Prints. In John T. Carpenter, ed., Hokusai and His Age: Ukiyo-e Painting, Printmaking, and Book Illustration in Late Edo Japan (Amsterdam: Hotei Publishing, 2005), pp. 234-69.

2. Leona, M. Microanalysis of organic pigments and glazes in polychrome works of art by surfaceenhanced resonance Raman scattering. Proc. Natl Acad. Sci. USA 106, 14757-14762 (2009).

3. Fukunaga, K. THz Technology applied to Cultural Heritage in Practice, Springer (2016).

4. Cheung, C. S., Daniel, J. M. O., Tokurakawa, M., Clarkson, W. A. \& Liang, H. High resolution Fourier domain optical coherence tomography in the $2 \mu \mathrm{m}$ wavelength range using a broadband supercontinuum source. Opt. Express, 23, 1992-2001 (2015). 
5. Read, M., Cheung, C.S., Liang, H., Meek, A., Korenberg, C. A Non-invasive Investigation of Egyptian Faience Using Long Wavelength Optical Coherence Tomography (OCT) at $2 \mu \mathrm{m}$. Studies in Conservation doi: 10.1080/00393630.2020.1871208 (2021).

6. Bonelli, N., Montis, C., Mirabile, A., Berti, D. \& Baglioni, P. Restoration of paper artworks with microemulsions confined in hydrogels for safe and efficient removal of adhesive tapes. Proc. Natl Acad. Sci. USA 115, 5932-5937 (2018).

7. Mastrangelo, R. et al. Twin-chain polymer hydrogels based on poly(vinyl alcohol) as new advanced tool for the cleaning of modern and contemporary art. Proc. Natl Acad. Sci. USA 117, 7011-7020 (2020).

8. Festa, G. \& Kardjilov, N. (Editors), Neutron Methods for Archaeology and Cultural Heritage, Springer International Publishing AG (2016).

9. Festa, G., et al. Egyptian grave goods of Kha and Merit studied by neutron and gamma techniques. Angew. Chem. Int. Ed. 57, 7375-7379 (2018).

10. Yang, B. \& Smith, A.M. Conventionally heated microfurnace for the graphitization of microgramsized carbon samples. Radiocarbon. 59 859-873 (2017).

11. Finch, D. et al. Ages for Australia's oldest rock paintings. Nature Human Behaviour 5, 310-316 (2021).

12. Cheung, C.S et al. Ultra-high resolution Fourier domain optical coherence tomography for old master paintings. Optics Express 23, 10145-10157 (2015).

13. Festa, $\mathrm{G}$ et al. Neutrons for Cultural Heritage-Techniques, Sensors, and Detection. Sensors 20, 502 (2020).

14. Jones, T. et al. Radiocarbon age constraints for a Pleistocene-Holocene transition rock art style: The Northern Running Figures of the East Alligator River region, western Arnhem Land, Australia. Journal of Archaeological Science: Reports 11, 80-89 (2017).

\section{Biographies}

Marco Leona is the David H. Koch Scientist in Charge of the Department of Scientific Research at The Metropolitan Museum of Art. He studied chemistry at the Università degli Studi di Pavia (Italy) where he obtained a Ph.D. in Mineralogy and Crystallography in 1995. Dr Leona's main research interest is the application of surface enhanced Raman spectroscopy to the study of pigments and dyes.

Kaori Fukunaga joined National Institute of Information and Communications Technology in 1994, and is the Director General of Applied Electromagnetic Research Center. She has been involved in deterioration analysis and nondestructive testing by using electromagnetic waves. She received her PhD in electrical engineering and BA in arts and design.

Haida Liang is Distinguished Professor of Physics and Head of the Imaging and Sensing for Archaeology, Art history and Conservation (ISAAC) Lab at Nottingham Trent University. She obtained a PhD in Astronomy and Astrophysics from the Australian National University studying clusters of galaxies using the Australia Telescope Compact Array (a radio interferometric array that is related to another type of interferometer invented by Michelson, the Michelson Stellar Interferometer). 
Piero Baglioni is Professor of Physical Chemistry at the University of Florence and is a MIT affiliate. He is on the editorial/advisory boards of several international journals and a member of several national and international institutions and societies. He is the author of more than 500 publications and 27 patents in the field of colloids and interfaces and a pioneer in the application of soft matter physics to the conservation of cultural heritage.

Giulia Festa is an experimental physicist whose research focuses on the development of neutron instrumentation and the analysis of materials applied to cultural heritage, collaborating with several international institutions and researchers worldwide. She has been Principal Investigator of more than 30 neutron experiments. Festa is co-editor of the first monograph about neutron methods applied to archaeology and cultural heritage.

Vladimir Levchenko is a Physicist with more than 35 years' experience in cosmogenic radionuclides and nearly 30 years' involvement with accelerator mass spectrometry (AMS). He joined ANSTO in 2006 and took a leading role in radiocarbon AMS in the range of applied studies extending from biomedical and ice core research to rock art, including Australian Aboriginal Rock Art research programs. Vladimir participated in a series of scientific expeditions for sample collection to remote areas, including Andes Altiplano in Bolivia, Kimberley and Arnhem Land in Australia.

\section{Acknowledgements}

ML makes the following acknowledgements: "I'd like to acknowledge Henry D. Smith II at Columbia University for his insights into the use and meaning of color in Japan, John Delaney, at the National Gallery of Art for his help with hyperspectral imaging, John Twilley, for teaching me everything I know about art analysis but certainly not everything he knows, the late John Winter, at the Freer Gallery of Art for introducing me to the study of Japanese art, and the late Richard Ernst, for countless discussions on art and science, and for waiting patiently, after I explained Fourier Transforms to him, before telling me about his research on Fourier Transform NMR, and that he was given a prize in Stockholm for that work."

KF would like to express sincere gratitude to the staff of the museums, especially conservators, for their cooperation and for their comments, which have been essential for the interpretation of the data.

HL gratefully acknowledges collaborations with scientists, conservators and curators in various museums, galleries and other heritage organisations, contributions made by past and present staff and students of the ISAAC Lab on OCT for art research in general, and in particular, C. S. Cheung for developing the 2 micron OCT, University of Southampton colleagues Andrew Clarkson, Masaki Tokurakawa and Jae Daniel for developing broad band lasers at 2 microns, and funding from the following: Leverhulme Trust Research Grant (F01 374F); UK Arts and Humanities Research Council and Engineering and Physical Sciences Research Council (AHRC/EPSRC AH/H032665/1); UK Arts and Humanities Research Council (AHRC CDP/National Gallery AH/R00174X/1); UK Arts and Humanities Research Council (AHRC CDP/British Museum AH/R001413/1); UK Arts and Humanities Research Council and Engineering and Physical Sciences Research Council (AHRC/EPSRC/English Heritage CDA08/429); UK Engineering and Physical Sciences Research Council (EPSRC/National Gallery CASE/CAN/04/90/). 
PB is indebted to all the conservators and students that enthusiastically contributed to the development of the field, and acknowledges the European Commission for its financial support of this exotic research field.

GF would like to thank all co-authors and researchers she had the opportunity to collaborate with in the field of multidisciplinary cultural heritage studies, for the fruitful collaborations and exchanges.

VL and the Rock Art studies program at the Centre of Accelerator Science at ANSTO acknowledge the financial support through the Australian National Collaborative Research Infrastructure Strategy and Rock Art Australia Foundation as well as help of the radiocarbon laboratory staff. He also would like to thank all co-authors and collaborators in the labs and in the field for the chance to participate in such exciting studies.

\section{Competing interests}

The authors declare no competing interests.

Figure 1. Artworks and cultural heritage that have been studied using physics methods. al Under the Wave off Kanagawa (Kanagawa oki nami ura), also known as The Great Wave, by Hokusai (circa 183032). b| "Irises at Yatsuhashi" by Ogata Korin (after 1709). c| The Madonna and Child (NG929, after Raphael, probably before 1600). d| Section of a wood panel representing the Virgin with the Child. el Sealed ceramic vase from grave goods of the ancient Egyptian architect Kha and his wife Merit (14251353 BCE). f| Rock art motifs stylistically classified as Northern Running Figures known to be early Holocene in age [14] from Manilakarr Clan Estate, western Arnhem Land, Northern Territory, Australia. Image reproduction permission kindly provided by Senior Traditional Owner Mr Alfred Nayinggul. Part a reproduced courtesy of H. O. Havemeyer Collection, Bequest of Mrs. H. O. Havemeyer, 1929. Part b adapted from Ref. [3], Springer Nature Limited. Part c adapted with permission from Ref. [12] (C) The Optical Society. Part d, image courtesy of Aurelia Chevalier. Part e is adapted from Ref. [13], CC BY 4.0. Part f image courtesy of Tristen Jones. 